\title{
Spatial maps in frontal and prefrontal cortex
}

\author{
Donald J. Hagler Jr. and Martin I. Sereno* \\ Department of Cognitive Science, University of California, San Diego, 9500 Gilman Dr. \#0515, La Jolla, CA 92093-0515, USA
}

Received 20 April 2005; revised 22 July 2005; accepted 2 August 2005

Available online 11 November 2005

Though the function of prefrontal cortex has been extensively investigated, little is known about the internal organization of individual prefrontal areas. Functional magnetic resonance imaging was used to show that some frontal and prefrontal cortical areas represent visual space in orderly, reproducible, topographic maps. The map-containing areas partly overlap dorsolateral prefrontal areas engaged by working memory tasks. These maps may be useful for attending to task-relevant objects at various spatial locations, an aspect of the executive control of attention.

(c) 2005 Elsevier Inc. All rights reserved.

\section{Introduction}

The prefrontal cortex has often been implicated in working memory processes. Several more specific functions have been proposed for this region, including maintenance and manipulation of information in working memory (Goldman-Rakic, 1987; Owen et al., 1996), attentional control (Corbetta et al., 1998), choosing between alternative responses (Rowe et al., 2000), and representation and storage of the rules of a task (Miller et al., 2002; Derrfuss et al., 2004). The neuronal circuitry required to implement these functions is not yet well understood.

Mammalian brains represent visual space in multiple retinotopic visual cortical areas. Though all of the maps in V1, V2, V3, $\mathrm{VP}, \mathrm{V} 4 \mathrm{v}$, and $\mathrm{V} 3 \mathrm{~A}$ represent all or part of the same contralateral visual hemifield, each area provides a unique mixture of preferences for particular stimulus properties. The topology of visual space is preserved to some degree in these maps, in that neighboring patches of cortex represent neighboring parts of the visual field, forming continuous maps across the cortical surface. A similar mapping of contralateral visual space across the cortical surface was recently revealed in the putative human lateral intraparietal area (LIP), located in posterior parietal cortex (Sereno et al., 2001). Unlike the maps in many earlier visual areas, however, this map only became evident when the subject was required to engage attention and working memory.

\footnotetext{
* Corresponding author. Fax: +1 8585341128.

E-mail address: sereno@cogsci.ucsd.edu (M.I. Sereno).

Available online on ScienceDirect (www.sciencedirect.com).
}

Working memory tasks also activate prefrontal cortex. Physiological recordings in monkeys have demonstrated that prefrontal areas have spatially selective memory fields that primarily represent restricted regions of contralateral space (Funahashi et al., 1989; Rainer et al., 1998). It seems plausible that maps of visual space might be preserved, rather than scrambled, as posterior visual areas project to frontal and prefrontal cortex. Indeed, Sawaguchi and Iba have recently identified one or more spatial maps in monkey dorsolateral prefrontal cortex (DLPFC) related to working memory and attention using delayed saccade paradigms (Sawaguchi and Iba, 2001). These maps may primarily be used to hold locations in memory for spatial working memory tasks or they might be more generally useful for a variety of tasks that require the allocation of working memory and attention. The homologies between monkey and human frontal and prefrontal areas are not completely clear, and so the relationship between these macaque monkey prefrontal maps and working-memory-related dorsolateral prefrontal areas in humans remains to be clarified.

In this paper, we ask whether working-memory-related areas in human DLPFC contain neurons with preferences for working memory stimuli appearing at particular spatial locations, and if so, whether these neurons are arrayed in a topological map of visual space. To address this question, we had subjects perform a working memory task with peripherally displayed images, steadily varying the polar angle of presentation. We have found that at least two of the frontal and prefrontal areas active during this task contain such maps of visual space.

The $n$-back working memory task is conceptually simple, yet it requires a complex set of mental functions to perform. A subject monitors for instances in which a stimulus is repeated after $n-1$ intervening stimuli. This task requires attention to stimulus features and feature comparison, the encoding and retrieval of stimuli in working memory, the maintenance, temporal ordering, and refreshing of working memory contents, and finally - when an overt response such as a button press is required - the choice, initiation, and sometimes suppression of motor responses. These subtasks require the cooperation of several cortical areas known to be involved in aspects of attention, working memory, and motor planning, including the frontal eye fields (FEF), posterior parietal cortex (PPC), DLPFC, and premotor cortex (Simon et al., 2002). 


\section{Materials and methods}

\section{Participants}

A total of 21 adults participated in this study ( 7 women). The mean age was $25 \pm 7$ years (ranging from 19 to 47). All subjects were right handed and had normal or corrected to normal vision. The experimental protocol was approved by the UCSD internal review board, and informed consent was obtained from each participant. There were two types of experimental designs used in this study, block design and phase-encoded. Twelve subjects participated in the block design experiment, and 20 participated in the phase-encoded experiment, with 11 subjects participating in both experiments. Before scanning, subjects were allowed, if they desired, to consume caffeinated beverages in order to better maintain alertness during the scan session.

\section{fMRI scanning}

A Varian 4 T or a GE 3 T scanner was used with a head coil, a single small surface coil, or a whole-head array of four or eight small surface coils (Nova Medical, Wakefield MA; General Electric). See Supplementary Table 1 for individual scan information for each subject. An echo planar T2*-weighted gradient echo pulse sequence $\left(8^{\prime} 32^{\prime \prime}\right.$ scan time, TR $=2000$ or $4000 \mathrm{~ms}$ for phase-encoded, $8^{\prime} 15^{\prime \prime}$ scan time, TR $=3000 \mathrm{~ms}$ for block design, $\mathrm{TE}=26.3 \mathrm{~ms}$, flip angle $=90^{\circ}$, bandwidth $=125 \mathrm{kHz}, 64 \times 64$ matrix, 21-36 axial slices, $3 \times 3 \times 3 \mathrm{~mm}$ or $3.75 \times 3.75 \times 3.8$ $\mathrm{mm}$ voxels) was used for functional scans. A T1-weighted MPRAGE scan $\left(\mathrm{TR}=10.5 \mathrm{~ms}, \mathrm{TE}=4.8 \mathrm{~ms}\right.$, flip angle $=11^{\circ}$, bandwidth $=50 \mathrm{kHz}, 256 \times 256$ matrix, 84 axial slices, $1 \times 1 \times 1.5$ $\mathrm{mm}$ voxels) was also acquired during each session to align the functional images to a previously obtained $(1.5,3$, or $4 \mathrm{~T})$ high resolution $(1 \times 1 \times 1 \mathrm{~mm})$ T1-weighted MPRAGE scan. Subjects' heads were immobilized with subject-specific dental impression bite-bars supported by a 4-ball-joint yoke. A custom image display program, run on a Silicon Graphics O2, was used to present images $\left(\sim 5^{\circ}\right.$ wide $\times \sim 6^{\circ}$ high $)$ centered at $\sim 8^{\circ}$ eccentricity. Images were projected onto a screen over the subject's ribcage using a standard video projector with a 7.38-12.3" focal length Xtra Bright Zoom lens (Buhl Optical, USA). Subjects viewed the screen indirectly via a mirror above the eyes.

\section{Stimuli}

Gray-scale images of 68 different faces were selected from the FERET database (Phillips et al., 1998), excluding those with facial hair or glasses. Images were closely cropped and then scaled to have identical vertical extent. Images were presented for $1.5 \mathrm{~s}$ each. Before each $23 \pm 3$ s block, a $2 \mathrm{~s}$ text message indicated whether the task for the next block would be identity, location, or passive. Block order was pseudorandom. Subjects indicated whether or not a 2-back match had occurred by right-handed button press (Photon Control Inc, Barnaby, B.C. Canada), with index finger $=$ no and middle finger $=$ yes. For the passive condition, subjects pressed the "no" button every time an image appeared. Images were not repeated except for 2-back matches until all other images had been presented. Potential 1-back and 3-back matches, and overlapping 2-back's were omitted to simplify the task. Frequency of matches was controlled by shuffling a list of the number of trials $(3,3,4,4$, $5,6)$ between matches. The likelihood of matches - either identity or location - for the identity blocks was identical to that for location blocks. The number of randomly chosen locations, eight, was also identical for all three block types. Simultaneous identity and location matches were disallowed to reduce the likelihood that subjects would focus on the identity of the face during a location block or vice versa. To prevent spontaneous recognition of matches, no 2-back matches of any kind occurred during the passive blocks. Additional tests outside the scanner with an optical eye-tracker confirmed that several subjects included in this study were able to maintain precise, reliable, central fixation during these tasks.

\section{Analysis of block design fMRI experiments}

Distortion in images was reduced with an image-based, B0-map correction method (Reber et al., 1998). Volume-registered block design experiment images were analyzed using AFNI's (Analysis of Functional NeuroImages) (Cox, 1996) 3dDeconvolve (Ward, B.D., Medical College of Wisconsin, 1998). A quadratic polynomial was used to fit the baseline. Motion estimates from 3 dvolreg were added to the baseline model to further reduce the contribution of motion to activation patterns. Correlation coefficients and $F$ statistics were generated for the area under the hemodynamic response function, which freely varied over $15 \mathrm{~s}$. For each subject in the group analysis, their cortical white matter surface was inflated to a sphere and warped into a best-fit sulcal alignment with an average of 40 spherical surfaces using FreeSurfer (Fischl et al., 1999). After resampling the deconvolution coefficients onto the average spherical surface, 10 steps of surfacebased smoothing were performed, equivalent to a $4 \mathrm{~mm}$ full-width, half-maximum (FWHM) Gaussian filter (Hagler et al., in preparation). AFNI's 3 dttest was used to generate $t$ statistics and means for each condition and the identity vs. location contrast. To facilitate viewing, results were sampled back onto the surface of a single subject. Cluster size exclusion was used for multiple comparison correction (Forman et al., 1995), with $t$ statistics thresholded at $P<0.05$ and surface clusters smaller than 145 contiguous vertices excluded. A cortical-surface-based program adapted from AFNI's AlphaSim (Ward, 2000) was used to estimate that these thresholds result in a corrected $P$ value of 0.05 (Hagler et al., in preparation). For comparison, group statistics were also generated from Talairach-transformed, $4 \mathrm{~mm}$ FWHM Gaussian smoothed, 3D volume data $(P<0.05$, uncorrected $)$.

\section{Analysis of phase-encoded fMRI experiments}

A Fourier transform of a time series generates a vector with real and imaginary components for each frequency that defines the amplitude and phase of periodic signals at that frequency. For activity at the stimulus frequency, the phase of this vector corresponds to the polar angle of the stimulus location. To estimate the significance of correlation of BOLD signal with the stimulus frequency, the squared amplitude of the signal at the stimulus frequency is divided by the sum of squared amplitudes at all other "noise" frequencies (excluding low frequency signals and harmonics of the stimulus frequency) (Sereno et al., 1995). This ratio of two chi-squared statistics follows the $F$ distribution (Larsen and Marx, 1986), and with degrees of freedom equal to the number of time points, this results in a statistical significance $P$ value. To increase SNR, multiple scans are averaged in the Fourier domain. The real and imaginary components are averaged across scans 
independently, which computes a vector average for each frequency. If the phases for a particular frequency are random, the amplitude will tend to be reduced to zero. Thus, the ratio between stimulus and noise frequencies will be maximal for those voxels displaying stimulus frequency activity with consistent phase and large amplitude. Subjects that displayed average data with widespread, high-amplitude periodic noise - probably due to heartbeat and respiration aliasing into the stimulus revolution frequency - across the majority of the cortical surface were excluded ( 5 of 20 subjects scanned). When possible, subjects were scanned with equal numbers of scans with counterclockwise or clockwise stimulus revolutions. Phases for clockwise data were reversed before averaging. 0.05 cycles of phase $(\sim 3 \mathrm{~s})$ were subtracted from the data before averaging to account for hemodynamic delays.

\section{Results}

Cortical areas activated during working memory tasks were first localized with block design functional magnetic resonance imaging (fMRI) experiments. Twelve subjects were scanned while perform-

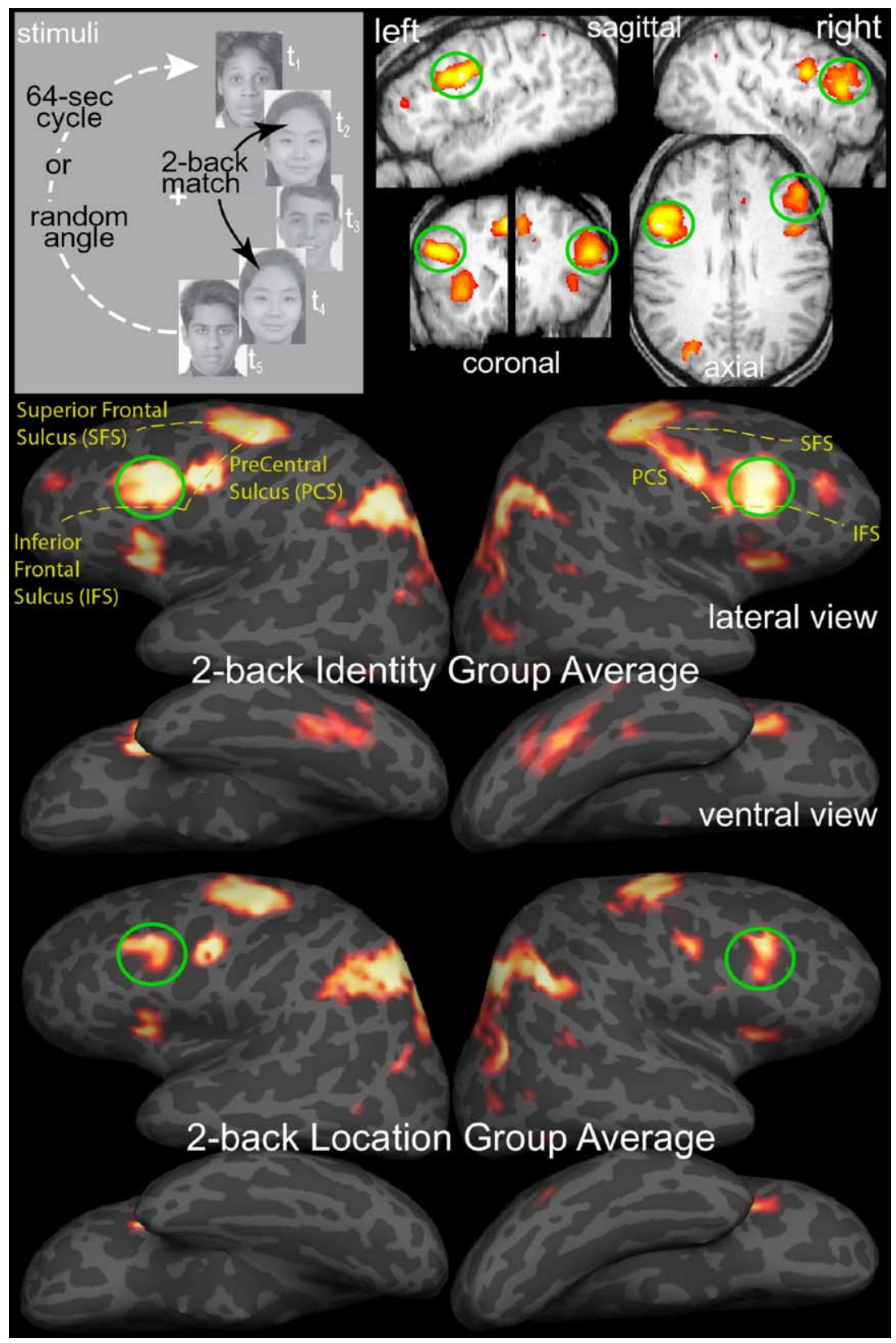

Fig. 1. 2-back face stimuli and task-related fMRI activations. Upper left, a schematized view of the 2-back face stimulus. White arrow represents the gradual translation of faces around the fixation cross; in the actual stimulus, only one face was presented at a time. For block design experiments, starting angle for each new face was pseudorandomly chosen. Upper right, planar views of a Talairach registered brain with superimposed, thresholded, group mean correlation coefficients from block design experiments with 2-back face identity task $(n=12$ subjects, $P<0.05$, uncorrected, volume-based $t$ test). Below are inflated, lateral, and ventral views of the reconstructed cortical surfaces showing areas of activation for the 2-back face identity and location tasks $(n=12$ subjects, $P<$ 0.05 , multiple comparison corrected, surface-based $t$ test). 
ing 2-back tasks. Subjects fixated centrally while viewing peripherally presented images of faces and pressed one button to indicate a 2-back match or another button to indicate no match. In the identity condition, subjects monitored for the repeated presentation of a particular face, regardless of its location. In the location condition, subjects monitored for repeated presentation of a face at one of eight pseudorandomly chosen locations, regardless of face identity. The identity and location blocks were compared with alternating passive blocks (randomized order) in which the subject viewed images of faces and pressed the same button each trial.
Widespread cortical activity was elicited by the 2-back face identity condition (Fig. 1). The focus of activation located in ventral temporal cortex is presumably the fusiform face area (FFA). Activity was not observed here for the 2-back face location task (Fig. 1, Supplementary Fig. 1), apparently reflecting a differential attention effect (Wojciulik et al., 1998). The focus in dorsolateral prefrontal cortex, highlighted in Fig. 1, sits in an area often implicated in working memory tasks, including the $n$-back task (Braver et al., 1997). Talairach coordinates for the approximate center of mass of this area in the left hemisphere were $-39,21,21$

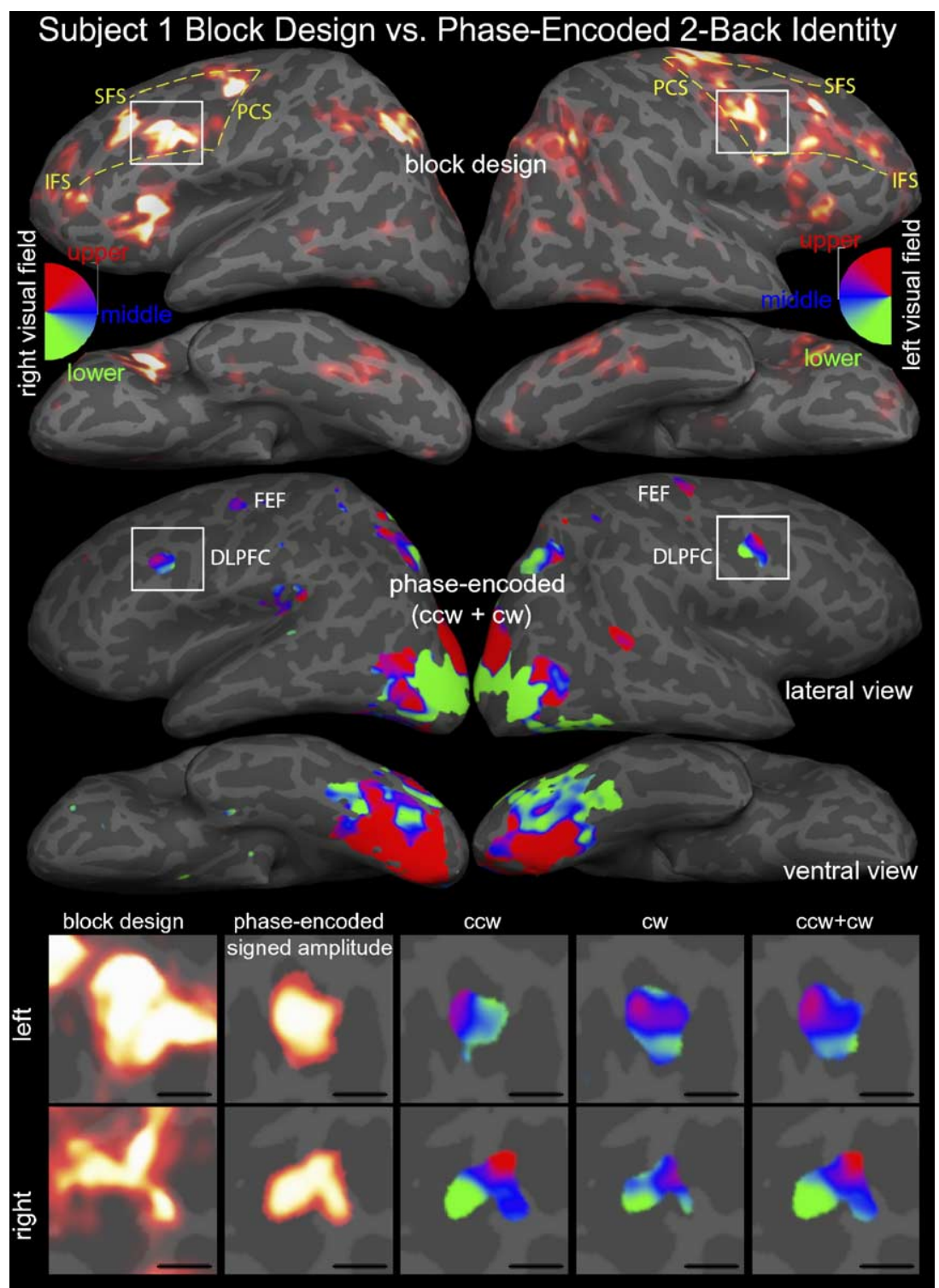

Fig. 2. Maps of visual space in a subset of working-memory-related areas. Results from a single subject (subject 1 in Fig. 4) are shown. Above are shown lateral and ventral views of the inflated left and right hemispheres comparing activations elicited using block design (top) or phase-encoded polar angle mapping (bottom) stimuli with the 2-back face identity task. Below are shown enlarged patches of flattened cortex from left (top row) and right (bottom row) prefrontal cortices. Corresponding areas on the lateral views above are marked with white bounding boxes. The leftmost column shows activations from the block design experiment. The second column shows the signed amplitude from Fourier analysis of phase-encoded mapping scans (average of 12 scans). Positive values (in orange) correspond to contralateral preference. Ipsilateral preference, if it were observed, would be represented with negative values and be displayed in blue. The third and fourth columns compare maps observed with counterclockwise or clockwise stimulus revolution directions, respectively (average of 6 scans each). The map color scheme for clockwise runs is reversed to make it consistent with counterclockwise runs. In both cases, red, blue, and green areas represent preference for upper, middle, and lower contralateral visual field, respectively. Last row shows maps from average of 12 scans (6 counterclockwise, 6 clockwise). Map data are thresholded at $P<0.05$ (Fourier analysis, uncorrected for multiple comparisons). Black scale bar in lower right corner of each enlarged ROI corresponds to $1 \mathrm{~cm}$. 
(3D average) and $-39,18,25$ (surface average); in the right hemisphere, they were 43, 28, 21 (3D average) and 38, 23, 24 (surface average). In both hemispheres, this area is less active during the 2-back location condition (Fig. 1, Supplementary Fig. 1); a paired $t$ test on the correlation coefficients within a cortical-surfacebased region of interest (ROI) confirmed that these task-related differences were statistically significant $(P<0.02)$. So as not to bias the result in favor of the identity task, the ROI for this comparison was defined based on the location task, which had a smaller area of activation than the identity task (Fig. 1). This difference in activation strength is possibly related to the greater difficulty of the 2-back identity task. Average percent correct was $73 \pm 17 \%( \pm \mathrm{SD})$ for 2-back identity matches and $84 \pm 8 \%$ for 2 back location matches ( $P=0.05$, paired $t$ test, $n=11$ subjects). Response times were also slower for the identity task $(0.81 \pm 0.13 \mathrm{~s}$ vs. $0.66 \pm 0.13 \mathrm{~ms}$., $P<10^{-5}$, paired $t$ test). A previous study, however, has shown that increased difficulty alone does not increase DLPFC activity (Barch et al., 1997; Heekeren et al., 2004). The increased activity may instead be due to additional working memory load or recruitment of neurons specialized for object working memory. At a minimum, it would appear that this area's function is not restricted to the storage of spatial locations (Owen et al., 1998).

Having localized the frontal and prefrontal areas active during a working memory task, we next tested for the existence of spatial maps in these areas using a phase-encoded stimulus design. The 2back face identity task was slightly modified for this purpose by smoothly and continuously varying the presentation location around the central fixation cross, with the identity of the face changing every $1.5 \mathrm{~s}$. A map is defined as an area displaying strong periodic activity at the stimulus revolution frequency (significantly greater than non-stimulus frequencies) with systematic and substantial spatial variation in response phase. Subjects performed the 2-back identity task continuously for the entire $512 \mathrm{~s}$ scan, with 8 complete revolutions per scan. It was apparently more difficult to perform this task continuously as the average percent correct (58 \pm $17 \%, n=19)$ was lower than for the block design version $(P=$ 0.03 , unpaired $t$ test; average response time $=0.78 \pm 0.80 \mathrm{~s}, P=$ 0.55 , unpaired $t$ test), although subjects still performed well above chance (chance level is the percentage of "yes" responses $=16 \pm$ $4 \%$, which is significantly different from percentage of correct matches; $P<10^{-5}$, paired $t$ test).

An example of one subject's mapping results is shown in Fig. 2 (also see Supplementary Fig. 2). As expected, maps were found in early visual areas and posterior parietal cortex. Maps were also found in a subset of the frontal and prefrontal areas recruited by the 2-back identity task. A newly discovered map was found in the inferior frontal sulcus, just anterior to the precentral sulcus (highlighted with a white bounding box). Another map was found in superior precentral sulcus (sup-PCS); this location corresponds to the FEF, an area involved in eye movements and visual attention (Paus, 1996; Corbetta et al., 1998; Beauchamp et al., 2001; Koyama et al., 2004). Note that the more anterior and inferior prefrontal foci active during the 2-back face identity task do not appear to contain spatial maps. The areas containing maps preferred contralateral stimulation; no frontal or prefrontal areas displayed a strong ipsilateral preference (Fig. 2). To verify that the

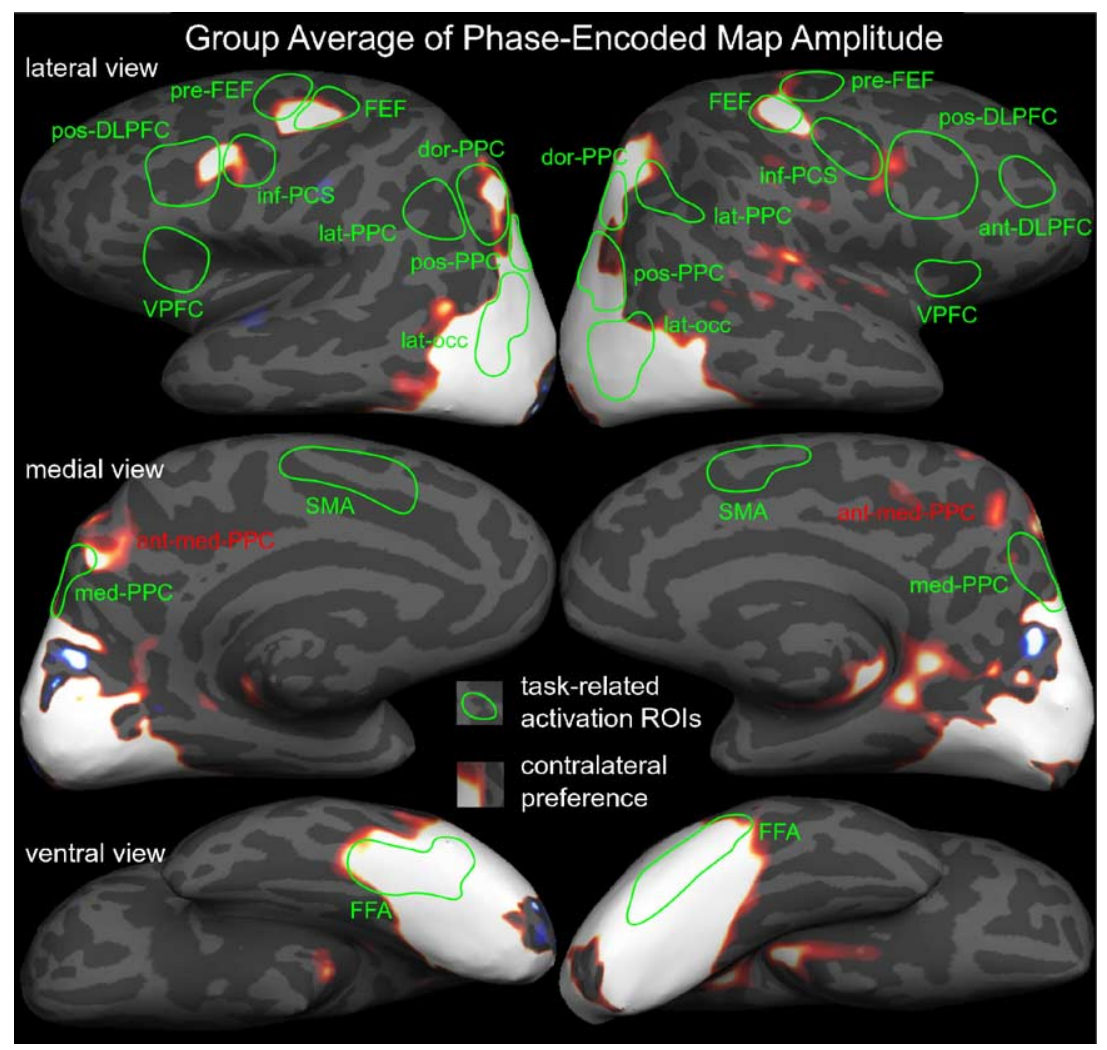

Fig. 3. Group average of contralateral preference during 2-back face mapping task. Inflated left and right cortical hemispheres with superimposed phaseencoded signed amplitude ( $n=12$ subjects). Lateral, medial, and ventral views are shown. Orange indicates positive values, or contralateral preference, whereas blue indicates negative, or ipsilateral preference. Outlines of the ROIs defined based on group average block design data are superimposed in green. $P$ values from ROI-based $t$ tests are shown in Table 1. 
phase-spread within these maps was not simply due to differences in hemodynamic delay, subjects were tested with both counterclockwise and clockwise stimulus revolution directions (Sereno et al., 1995, 2001). After reversing the phases of the clockwise runs to be consistent with counterclockwise runs, the resulting maps are quite similar, demonstrating that the phase-spreads are stimulusdriven (Fig. 2).

We have developed a simple method for group analysis of phase-encoded visuospatial mapping data. Single subject Fourier analysis yields two values, amplitude and phase, for each voxel or patch of cortical surface. The phase corresponds to the preferred segment of visual space. The amplitude reflects the statistical significance of the correlation with the stimulus revolution frequency and is equal to $-\log _{10}$ of the $P$ value (e.g. $\left.-\log _{10}(0.001)=3\right)$. Although the phase at a given patch of cortical surface may vary greatly between subjects - especially for small maps - the intersubject average of the amplitude can be used to identify areas that reliably display activity at the stimulus revolution frequency. To further refine this analysis, we set the amplitude positive or negative depending on whether the phase represents contralateral or ipsilateral space. The intersubject average of this signed amplitude can be used to identify areas with reliable contralateral preference. Average results from twelve subjects are shown in Fig. 3. The average amplitudes of contralateral preference are clearly highest in early visual areas of the occipital cortex, extending into the FFA. Importantly, peaks are also found in DLPFC, FEF, and PPC.

ROI-based analysis of these contralateral preference values confirmed the qualitative results shown in Fig. 3. Cortical surfacebased ROIs (as outlined in Fig. 3) were defined based on the supra-threshold foci $(P<0.05$, corrected for multiple comparisons) in the group mean of block design 2-back face identity data. These ROIs were then resampled onto the surface of each subject using sulcal alignment (see Materials and methods). The signed amplitudes of the phase-encoded mapping data were averaged within those ROIs and then averaged across subjects. A $t$ test was performed to identify ROIs with average contralateral preference amplitudes significantly different from zero (Table 1). Results for several ROIs reached significance, including the bilateral DLPFC foci in the posterior inferior frontal sulcus (pos-IFS), the bilateral FEF foci in sup-PCS, and bilateral inferior PCS (inf-PCS). Average values for nearby ROIs, such as right anterior DLPFC, bilateral ventral PFC, and left pre-FEF, were not significantly different from zero, indicating a lack of preference for either hemifield.

As an additional group analysis, we also counted the number of subjects displaying maps in each of these ROIs. In the posterior DLPFC ROI, 67\% (10/15) of subjects displayed maps in the left hemisphere, whereas $75 \%(9 / 12)$ of subjects (with coil coverage of right hemisphere) displayed maps in the right hemisphere. The percentages of subjects displaying visuospatial maps in various ROIs, as well as the average MNI Talairach coordinates for those maps, are summarized in Table 2. These cortical surface-based ROIs were defined based on the supra-threshold foci $(P<0.05$, corrected for multiple comparisons) in the group mean of block design face identity data. The numbers in Table 2 reflect data from fifteen subjects, four of whom were scanned with a single surface coil placed over the left frontal cortex in an attempt to improve the signal-to-noise ratio (SNR). Restricting the analysis to the twelve subjects with whole-head coverage (as in Fig. 3 and Table 1) allows us to determine the number of subjects with maps in either
Table 1

$P$ values from ROI-based $t$ test of contralateral preference

\begin{tabular}{|c|c|c|}
\hline Region of interest & Left hemisphere & Right hemisphere \\
\hline pos-DLPFC (pos-IFS) & $0.0098^{*}$ & 0.013* \\
\hline ant-DLPFC (ant-IFS) & NA & 0.56 \\
\hline VPFC (ant-lat-sulc) & 0.55 & 0.36 \\
\hline FEF (sup-PCS) & $0.0042 *$ & $0.0064 *$ \\
\hline pre-FEF (pos-SFS) & 0.076 & $0.021 *$ \\
\hline inf-PCS & $0.036 *$ & 0.028* \\
\hline pos-PPC & $0.024 *$ & $0.012 *$ \\
\hline dor-PPC & 0.0038* & 0.013* \\
\hline lat-PPC & 0.93 & 0.071 \\
\hline med-PPC & $0.0065 *$ & $0.040 *$ \\
\hline lat-occ & $0.0023 *$ & $0.00058^{*}$ \\
\hline SMA (cing) & $(-) 0.18$ & $(-) 0.81$ \\
\hline FFA (fus) & $0.00046^{*}$ & $0.0022 *$ \\
\hline
\end{tabular}

ROI-based $t$ tests of contralateral preference amplitudes. For each ROI, $P$ values are given from a one-sample two-tailed $t$ test on the signed amplitude of correlation with the phase-encoded stimulus revolution frequency. Only subjects with whole head scan coverage were included in this analysis $(n=12)$. Cortical surface ROIs, as shown in Fig. 3, were defined based on the block design 2-back face identity group average and resampled onto the cortical surface of each subject using sulcal alignment (see Materials and methods). Statistically significant $(P<0.05)$ values are shown in bold. ROIs with a negative mean (ipsilateral preference) are indicated with a "-" before the $P$ value. Abbreviations: anterior (ant), lateral (lat), posterior (pos), superior (sup), inferior (inf), dorsal (dor), medial (med), inferior frontal sulcus (IFS), precentral sulcus (PCS), superior frontal sulcus (SFS), lateral sulcus (lat-sulc), ventral prefrontal cortex (VPFC), posterior parietal cortex (PPC), supplementary motor area (SMA), cingulate (cing), fusiform face area (FFA), fusiform (fus).

$* P<0.05$.

or both hemispheres. For example, 100\% (12/12) had at least one map in either left or right pos-DLPFC, and 45\% (5/12) displayed maps in both left and right pos-DLPFC. 83\% (10/12) of subjects displayed maps in either left or right FEF (sup-PCS), and 42\% (5/ 12) displayed maps in both left and right FEF. Such comparisons for the other ROIs containing maps are shown in Table 3 (see also Supplementary Table 2). Frequently, maps were also found in some subjects in areas other than those active in the 2-back face identity task. Anterior medial PPC contains such an area, visible in the group average in Fig. 3 (medial view, labeled in red, just anterior to medial PPC ROI), containing maps in 53\% (8/15) and 67\% (8/12) of subjects in right and left hemispheres, respectively (see also Supplementary Table 2).

Maps from ten subjects (twelve hemispheres) are compared in Fig. 4. Although the general location of these maps is similar across subjects, there are differences in the number of detectable maps. There are also apparent intersubject differences in the orientation of each map (the gradient of the phase map with respect to position on the cortical surface). Some of these directional differences are less than $90^{\circ}$ and may simply reflect anatomical variation. Other differences, such as $180^{\circ}$ rotation or bifurcation, are not as easy to interpret as variations of the sort known from early visual areas. Although these variations could reflect real functional and anatomical differences between subjects, they could also result from measurement limitations. Sampling multiple adjoining maps with voxels that are not small enough could result in erroneous phase measurements. Different cortical folding patterns can also introduce artifacts given that standard fMRI voxels are comparable in width to the 
Table 2

Visuospatial maps found in foci activated by 2-back faces task

\begin{tabular}{|c|c|c|c|c|c|}
\hline Region of interest & Hemisphere & Subjects with maps & $x$ & $y$ & $z$ \\
\hline \multirow{2}{*}{ pos-DLPFC (pos-IFS) } & Left & $67 \%(10 / 15)$ & $-36 \pm 5$ & $16 \pm 3$ & $28 \pm 4$ \\
\hline & Right & $75 \%(9 / 12)$ & $40 \pm 4$ & $23 \pm 11$ & $24 \pm 9$ \\
\hline ant-DLPFC (ant-IFS) & Right & $8 \%(1 / 12)$ & 42 & 46 & 15 \\
\hline \multirow[t]{2}{*}{ VPFC (ant-lat-sulc) } & Left & $7 \%(1 / 15)$ & -37 & 29 & 3 \\
\hline & Right & $0 \%(0 / 12)$ & & & \\
\hline \multirow[t]{2}{*}{ FEF (sup-PCS) } & Left & $73 \%(11 / 15)$ & $-36 \pm 5$ & $-13 \pm 5$ & $50 \pm 3$ \\
\hline & Right & $58 \%(7 / 12)$ & $37 \pm 7$ & $-9 \pm 3$ & $48 \pm 5$ \\
\hline \multirow[t]{2}{*}{ pre-FEF (pos-SFS) } & Left & $40 \%(6 / 15)$ & $-31 \pm 4$ & $-5 \pm 5$ & $49 \pm 5$ \\
\hline & Right & $42 \%(5 / 12)$ & $30 \pm 6$ & $-5 \pm 6$ & $53 \pm 5$ \\
\hline \multirow[t]{2}{*}{ inf-PCS } & Left & $47 \%(7 / 15)$ & $-41 \pm 6$ & $0 \pm 4$ & $32 \pm 5$ \\
\hline & Right & $42 \%(5 / 12)$ & $41 \pm 5$ & $2 \pm 7$ & $30 \pm 7$ \\
\hline \multirow{2}{*}{ pos-PPC } & Left & $67 \%(10 / 15)$ & $-23 \pm 4$ & $-79 \pm 3$ & $32 \pm 5$ \\
\hline & Right & $83 \%(10 / 12)$ & $29 \pm 4$ & $-73 \pm 4$ & $26 \pm 5$ \\
\hline \multirow[t]{2}{*}{ dor-PPC } & Left & $47 \%(7 / 15)$ & $-27 \pm 6$ & $-62 \pm 9$ & $52 \pm 7$ \\
\hline & Right & $67 \%(8 / 12)$ & $30 \pm 5$ & $-61 \pm 6$ & $52 \pm 3$ \\
\hline \multirow[t]{2}{*}{ lat-PPC } & Left & $7 \%(1 / 15)$ & -22 & -48 & 50 \\
\hline & Right & $25 \%(3 / 12)$ & $28 \pm 7$ & $-48 \pm 1$ & $49 \pm 1$ \\
\hline \multirow[t]{2}{*}{ med-PPC } & Left & $40 \%(6 / 15)$ & $-9 \pm 5$ & $-72 \pm 6$ & $50 \pm 6$ \\
\hline & Right & $42 \%(5 / 12)$ & $12 \pm 10$ & $-67 \pm 8$ & $47 \pm 7$ \\
\hline \multirow[t]{2}{*}{ lat-occ } & Left & $93 \%(14 / 15)$ & $-33 \pm 6$ & $-83 \pm 5$ & $15 \pm 6$ \\
\hline & Right & $92 \%(11 / 12)$ & $42 \pm 5$ & $-78 \pm 6$ & $7 \pm 6$ \\
\hline \multirow[t]{2}{*}{ SMA (cing) } & Left & $0 \%(0 / 15)$ & & & \\
\hline & Right & $0 \%(0 / 12)$ & & & \\
\hline \multirow[t]{2}{*}{ FFA (fus) } & Left & $92 \%(11 / 12)$ & $-37 \pm 4$ & $-69 \pm 5$ & $-15 \pm 2$ \\
\hline & Right & $92 \%(11 / 12)$ & $41 \pm 5$ & $-71 \pm 7$ & $-12 \pm 4$ \\
\hline
\end{tabular}

Visuospatial maps found in foci activated by 2-back face task. Average MNI Talairach coordinates are given ( \pm SD) for the maps found in each ROI (with $P<$ 0.05 , Fourier analysis, uncorrected for multiple comparisons) defined based on block design 2-back face identity group average (as shown in Fig. 3). Also given are the percentages of subjects with maps. Only those subjects with scan coverage at a given location were included in those percentages (e.g. four subjects with left hemisphere coverage only were not included in right hemisphere map counts). In most cases, map coordinates are from the approximate center of the middle field representation. Often, however, ROIs contained what appeared to be multiple contiguous maps. Furthermore, as in the fusiform ROI, maps may be contiguous with other maps outside the ROI (i.e. early visual areas). In these cases, map coordinates given correspond to the approximate center of mass of the map(s) (or portions thereof) found inside the ROI. Percentages from ROIs with maps in a majority of subjects are shown in bold.

thickness of the cortex. More accurate maps could be obtained with considerably smaller voxel sizes; however, this will have to wait for the development of many-element phased-array coils to compensate for the greatly reduced SNR that results from using small voxels (since SNR is approximately proportional to

Table 3

Percentages of subjects with maps in either vs. both hemispheres

\begin{tabular}{lcc}
\hline Region of interest & Either hemisphere & Both hemispheres \\
\hline pos-DLPFC (pos-IFS) & $100 \%(12 / 12)$ & $42 \%(5 / 12)$ \\
ant-DLPFC (ant-IFS) & NA & NA \\
VPFC (ant-lat-sulc) & $0 \%(0 / 12)$ & $0 \%(0 / 12)$ \\
FEF (sup-PCS) & $83 \%(10 / 12)$ & $42 \%(5 / 12)$ \\
pre-FEF (pos-SFS) & $67 \%(8 / 12)$ & $17 \%(2 / 12)$ \\
inf-PCS & $67 \%(8 / 12)$ & $8 \%(1 / 12)$ \\
pos-PPC & $92 \%(11 / 12)$ & $58 \%(7 / 12)$ \\
dor-PPC & $75 \%(9 / 12)$ & $42 \%(5 / 12)$ \\
lat-PPC & $25 \%(3 / 12)$ & $8 \%(1 / 12)$ \\
med-PPC & $75 \%(9 / 12)$ & $17 \%(2 / 12)$ \\
lat-occ & $100 \%(12 / 12)$ & $92 \%(11 / 12)$ \\
SMA (cing) & $0 \%(0 / 12)$ & $0 \%(0 / 12)$ \\
FFA (fus) & $100 \%(12 / 12)$ & $83 \%(10 / 12)$ \\
\hline
\end{tabular}

Numbers of subjects with maps in either vs. both hemispheres. For each ROI, the percentages of subjects with maps in either hemisphere or both hemispheres are given. Subjects included in these counts were those with whole head scan coverage $(n=12)$. voxel volume, $2 \times 2 \times 2 \mathrm{~mm}$ voxels have less than $1 / 3$ the SNR of $3 \times 3 \times 3 \mathrm{~mm}$ voxels).

Six subjects were scanned on multiple occasions to verify that these maps were reproducible within subjects, circumventing the between-subject variability due to inadequate spatial resolution and differences in cortical folding. One such subject was scanned four times on each of three occasions for a total of twelve scans (Fig. 5). The location of the periodically activated prefrontal area and the direction of phase change are preserved in each of the three session averages. The small changes across sessions $(\sim 1 \mathrm{~mm})$ are within the measurement errors inherent in aligning lower resolution functional images with higher resolution structural images used to make the cortical surface reconstruction. Similar map repeatability between sessions was observed for three other subjects.

\section{Discussion}

Although not explicitly requiring spatial working memory, the 2-back face identity task, used here to demonstrate the existence of maps in multiple frontal areas, requires attention to objects in a particular spatial location. Other areas involved in working memory may use the DLPFC maps we have found to allocate attention and perform operations on objects at particular locations. The 2-back location task, which explicitly requires storage of spatial locations, actually activated DLPFC less strongly in block 


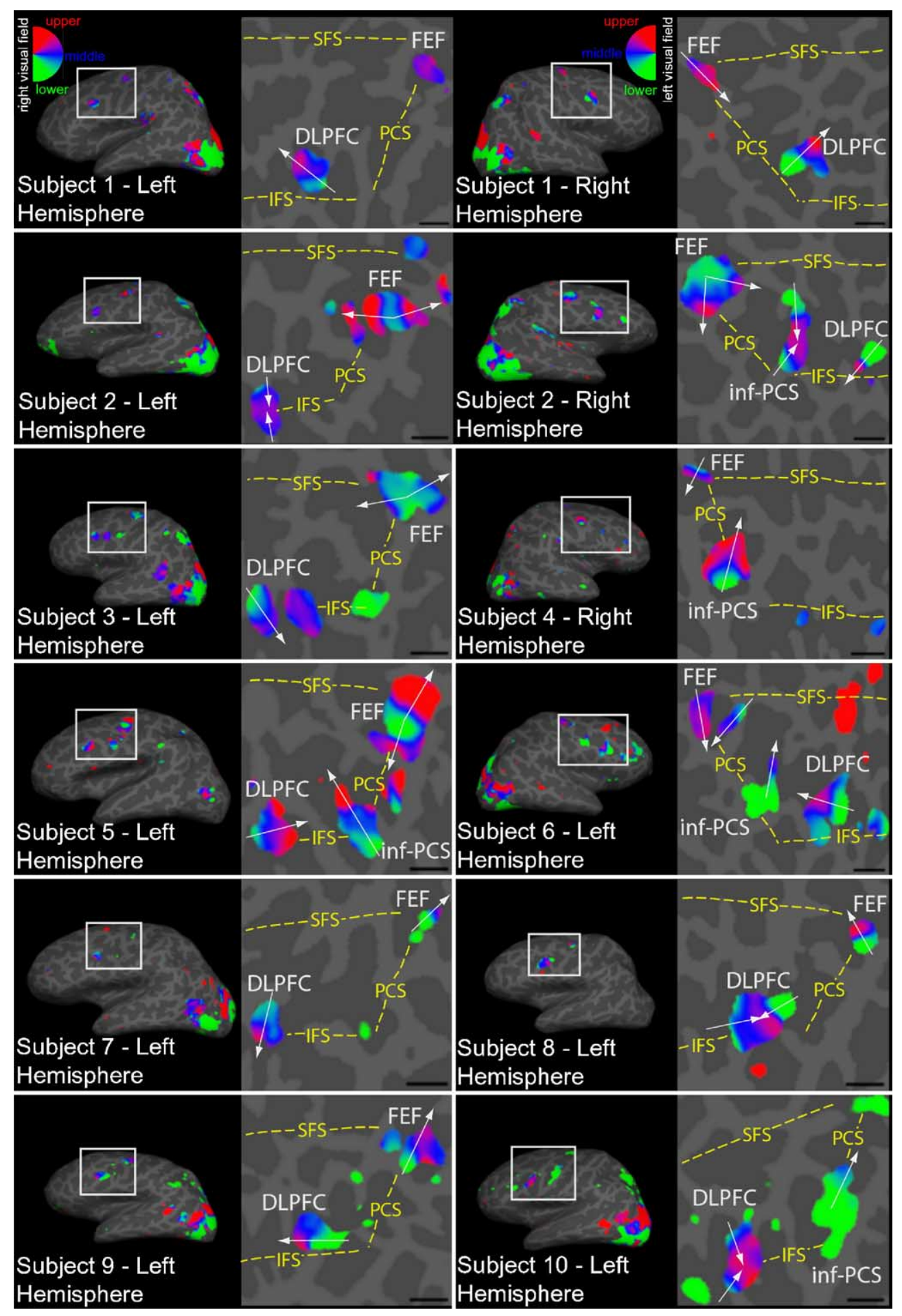

Fig. 4. Spatial maps in frontal and prefrontal cortex in multiple subjects. Patches of flattened frontal/prefrontal cortex from nine left hemispheres and three right hemispheres (10 subjects) with superimposed phase-map statistics from scans acquired with the phase-encoded 2-back face stimulus. Each shows the average of 2-12 scans acquired over 1-3 sessions (see Supplementary Table 1). Adjacent images show the same data on the inflated cortical surface of each subject. Subject 1 is same subject as in Figs. 2 and 5. White arrows represent the estimated direction of phase change within a given polar angle map (lower to upper field). Red, blue, and green areas represent preference for upper, middle, and lower contralateral visual field, respectively. Map data are thresholded at $P<0.05$ (Fourier analysis, uncorrected for multiple comparisons). Scale bars correspond to $1 \mathrm{~cm}$.

design experiments (Fig. 1); nonetheless, it is possible that the location task also recruits these maps. We were unable to directly test this, however, as the predictable spatial locations of the phaseencoded mapping method make a 2-back location task impossible.
We had initially attempted to uncover prefrontal maps using a delayed saccade task. This spatial working memory task has been used extensively in studies of monkeys (Funahashi et al., 1989; Sawaguchi and Iba, 2001) and humans (Sweeney et al., 1996), 


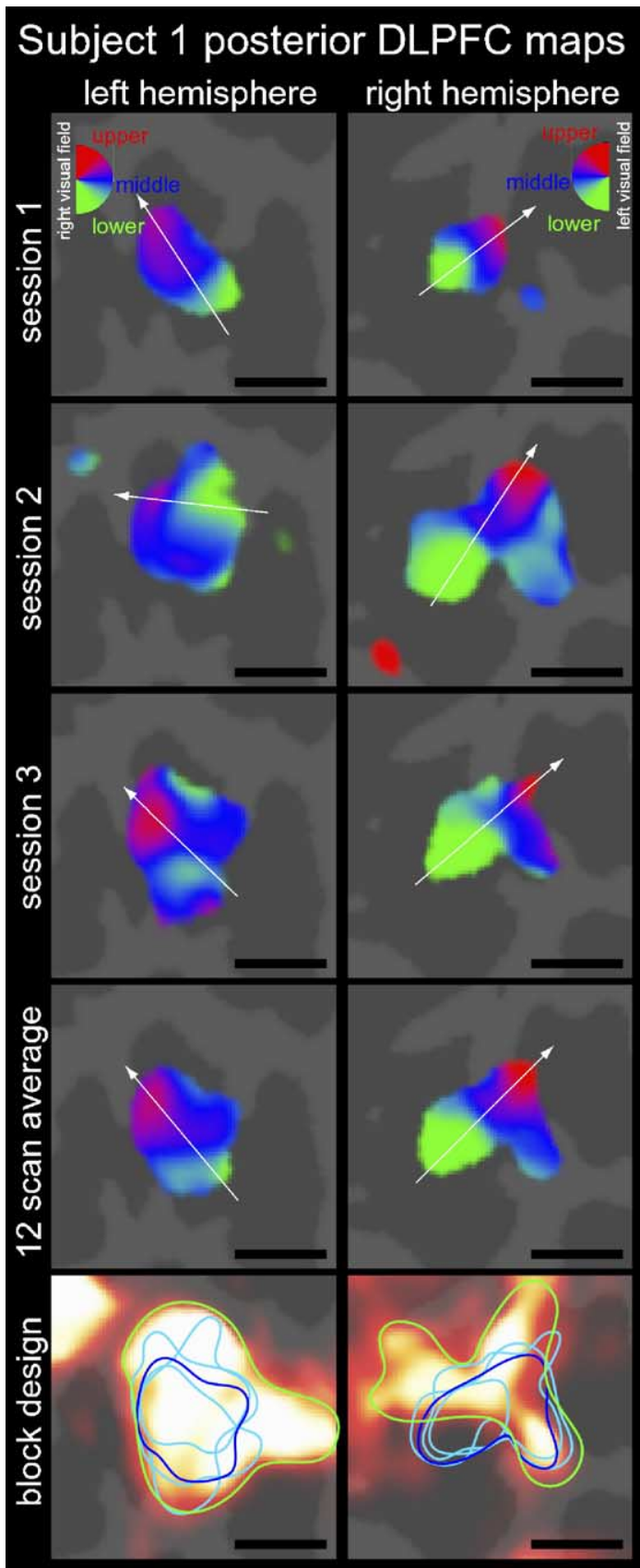

Fig. 5. Spatial maps in frontal and prefrontal cortex observed repeatedly in a single subject (Subject 1 in Fig. 4). Left and right columns show patches of flattened cortex from left and right prefrontal cortices, respectively, with superimposed phase-map statistics from scans acquired with the phaseencoded 2-back face stimulus (identical ROI as in Fig. 2). The first three rows show the four-scan average for each of three separate sessions (acquired over a 2-week period). The fourth row shows the twelve-scan cross-session average. Red, blue, and green areas represent preference for upper, middle, and lower contralateral visual field, respectively. Map data are thresholded at $P<0.05$ (Fourier analysis, uncorrected for multiple comparisons). White arrows represent the estimated direction of phase change within a given polar angle map. In the bottom panels are the same patches of cortex with thresholded correlation coefficients obtained from this particular subject with the block design stimulus $\left(P<10^{-5}\right.$, uncorrected for multiple comparisons). Outlines drawn show the borders of the map areas (blue for the cross-session average, cyan for the session averages, and green for block design). Scale bars correspond to $1 \mathrm{~cm}$. including an fMRI study that identified a map in the putative human LIP (Sereno et al., 2001). Subjects were briefly presented with a small dot at one of eight peripheral locations. After a short delay, the subject made a saccade to the remembered location and then returned gaze back to the central fixation cross. Despite robust parietal and superior frontal activity, relatively little dorsolateral prefrontal activity was observed in block design experiments, and DLPFC maps could not be reliably demonstrated in phase-encoded experiments (Hagler et al., in preparation). This suggests that these DLPFC maps cannot be strongly activated by merely remembering a location; instead, they are interested in both location and content. In contrast, the pre-FEF, an area that has been shown to be specialized for spatial working memory (Courtney et al., 1998), displayed robust map activity (and contralateral preference) in our saccade mapping experiments (Hagler et al., in preparation); whereas with the 2-back identity task, the contralateral preference is largely confined to the FEF (Fig. 3).

The frontal maps showed considerable intersubject variability (Fig. 4, Tables 2 and 3, Supplementary Table 2). Previous imaging studies of retinotopic areas in occipital cortex - especially in areas beyond V1 and V2 - have also revealed variability in the precise location of areal boundaries and the degree to which maps are represented in their entirety (i.e. including upper, middle, and lower visual fields) (DeYoe et al., 1996; Hadjikhani et al., 1998; Huk et al., 2002). There are several reasons why maps in higherlevel areas are more difficult to accurately and reliably measure. First, in areas such as DLPFC, only a subset of neurons display spatial preference (Funahashi et al., 1989; Rainer et al., 1998). Second, task-related activity that is non-spatially selective could cause fluctuations in blood oxygenation level dependent (BOLD) signals that actively reduce the significance of the correlation with stimulus location; this effect could differ between hemispheres. Third, the maps we have found are considerably smaller than the maps in areas such as V1 and V2 and are, therefore, harder to detect. Finally, detecting maps in cortical areas involved in working memory and attention is more sensitive to how consistently a subject can maintain focus on the task.

Spatial maps related to eye movements, spatial attention, and working memory have been identified in monkey frontal and prefrontal cortex, in and around the FEF (Robinson and Fuchs, 1969; Suzuki and Azuma, 1983; Bruce et al., 1985) and principal sulcus (Sawaguchi and Iba, 2001; Roe et al., 2004). It has been argued that the human homologues of both the FEF and the principal sulcus area are located more superiorly than in monkeys (Courtney et al., 1998), suggesting that our DLPFC focus may be comparable to a more region inferior to the FEF and the principal sulcus in monkeys. The task we used to identify these new maps in human prefrontal cortex is more complicated in terms of mental components required than the simple spatial working memory tasks often used in monkey working memory studies; however, the 2-back task is essentially a continuously running version of the object-based match-to-sample task, which has been used successfully in monkeys (Rainer et al., 1998). In that study, prefrontal neurons - particularly those inferior to the principal sulcus - were found to display "memory fields"; that is, task-related activity with spatial preference. Perhaps, spatial maps in this region of monkey prefrontal cortex remain to be uncovered. We also found maps in the inf-PCS of several subjects. Recently, passive visual responses have been found in macaque monkeys between hand and face motor cortex on the precentral gyrus (Graziano and Gandhi, 2000). Our inf-PCS maps may be similar to that area. 
A variety of disparate tasks recruit human DLPFC (Duncan and Owen, 2000; Derrfuss et al., 2004). Although there is strong evidence of this area's involvement in working memory tasks (Goldman-Rakic, 1987; Funahashi et al., 1989; Owen et al., 1996; Braver et al., 1997; Rainer et al., 1998), some have suggested that the basic role of DLPFC is to maintain an abstract representation of the rules of a task (Miller et al., 2002; Derrfuss et al., 2004) and to select responses (Rowe et al., 2000). Our results suggest some degree of functional subdivision within DLPFC as the maps do not completely cover the posterior DLPFC region activated by our task (Figs. 2 and 3). DLPFC may also be multifunctional; that is, mapcontaining areas could also be involved in several distinct operations, such as task representation, attending to salient objects at various locations, maintaining information in working memory, and response selection.

Representing the rules of a task may simply be a special case of working memory. The contents of working memory are often viewed as an activated subset of information from long-term memory, with the focus of attention determining what information becomes active (Cowan, 1988; Engle et al., 1999). Such a model does not require that the attentional controller, perhaps DLPFC, actually represents the information. Instead, DLPFC may simply know which cortical areas to activate in order to access relevant information. These other areas could include ventral temporal areas encoding object features (Wojciulik et al., 1998), posterior parietal areas encoding stimulus-response relationships (Bunge et al., 2002), and areas more directly involved in movement. The visuospatial maps in DLPFC may index maps not only in parietal cortex but also in inferotemporal cortex, where we found apparent retinotopy in the FFA; this is in contrast to the conclusions of an earlier study, in which simpler stimuli were used for retinotopic mapping (Halgren et al., 1999). The function of DLPFC may be to coordinate the allocation of attention to task-relevant information, whether that information is stimulus location, image features, or the correct stimulusresponse relationship.

\section{Acknowledgments}

We would like to thank Y. Goda and members of the Sereno laboratory for helpful comments on early versions of the manuscript. We also thank R. Buxton, L.R. Frank, T.T. Liu, L. May, and E.C. Wong at the UCSD fMRI Center for development and support of scanner hardware, pulse sequences, and image reconstruction and correction software. This work was supported by an NSF grant BCS 0224321 to M.S. and an NIH NRSA fellowship to D.H.

\section{Appendix A. Supplementary data}

Supplementary data associated with this article can be found in the online version at doi:10.1016/j.neuroimage.2005.08.058.

\section{References}

Barch, D.M., Braver, T.S., Nystrom, L.E., Forman, S.D., Noll, D.C., Cohen, J.D., 1997. Dissociating working memory from task difficulty in human prefrontal cortex. Neuropsychologia 35, 1373-1380.

Beauchamp, M.S., Petit, L., Ellmore, T.M., Ingeholm, J., Haxby, J.V., 2001.
A parametric fMRI study of overt and covert shifts of visuospatial attention. NeuroImage 14, 310-321.

Braver, T.S., Cohen, J.D., Nystrom, L.E., Jonides, J., Smith, E.E., Noll, D.C., 1997. A parametric study of prefrontal cortex involvement in human working memory. NeuroImage 5, 49-62.

Bruce, C.J., Goldberg, M.E., Bushnell, M.C., Stanton, G.B., 1985. Primate frontal eye fields: II. Physiological and anatomical correlates of electrically evoked eye movements. J. Neurophysiol. 54, 714-734.

Bunge, S.A., Hazeltine, E., Scanlon, M.D., Rosen, A.C., Gabrieli, J.D., 2002. Dissociable contributions of prefrontal and parietal cortices to response selection. NeuroImage 17, 1562-1571.

Corbetta, M., Akbudak, E., Conturo, T.E., Snyder, A.Z., Ollinger, J.M., Drury, H.A., Linenweber, M.R., Petersen, S.E., Raichle, M.E., Van Essen, D.C., Shulman, G.L., 1998. A common network of functional areas for attention and eye movements. Neuron 21, 761-773.

Courtney, S.M., Petit, L., Maisog, J.M., Ungerleider, L.G., Haxby, J.V., 1998. An area specialized for spatial working memory in human frontal cortex. Science 279, 1347-1351.

Cowan, N., 1988. Evolving conceptions of memory storage, selective attention, and their mutual constraints within the human informationprocessing system. Psychol. Bull. 104, 163-191.

Cox, R.W., 1996. AFNI: software for analysis and visualization of functional magnetic resonance neuroimages. Comput. Biomed. Res. $29,162-173$.

Derrfuss, J., Brass, M., von Cramon, D.Y., 2004. Cognitive control in the posterior frontolateral cortex: evidence from common activations in task coordination, interference control, and working memory. NeuroImage 23, 604-612.

DeYoe, E.A., Carman, G.J., Bandettini, P., Glickman, S., Wieser, J., Cox, R., Miller, D., Neitz, J., 1996. Mapping striate and extrastriate visual areas in human cerebral cortex. Proc. Natl. Acad. Sci. U. S. A. 93, $2382-2386$.

Duncan, J., Owen, A.M., 2000. Common regions of the human frontal lobe recruited by diverse cognitive demands. Trends Neurosci. 23, 475-483.

Engle, R.W., Tuholski, S.W., Laughlin, J.E., Conway, A.R., 1999. Working memory, short-term memory, and general fluid intelligence. a latentvariable approach. J. Exp. Psychol. Gen. 128, 309-331.

Fischl, B., Sereno, M.I., Tootell, R.B., Dale, A.M., 1999. High-resolution intersubject averaging and a coordinate system for the cortical surface. Hum. Brain Mapp. 8, 272-284.

Forman, S.D., Cohen, J.D., Fitzgerald, M., Eddy, W.F., Mintun, M.A., Noll, D.C., 1995. Improved assessment of significant activation in functional magnetic resonance imaging (fMRI): use of a cluster-size threshold. Magn. Reson. Med. 33, 636-647.

Funahashi, S., Bruce, C.J., Goldman-Rakic, P.S., 1989. Mnemonic coding of visual space in the monkey's dorsolateral prefrontal cortex. J. Neurophysiol. 61, 331-349.

Goldman-Rakic, P.S., 1987. Circuitry of primate prefrontal cortex and regulation of behavior by representational memory. In: Plum, F., Mountcastle, F. (Eds.), Handbook of Physiology. The American Physiological Society, Washington, DC, pp. 373-517.

Graziano, M.S., Gandhi, S., 2000. Location of the polysensory zone in the precentral gyrus of anesthetized monkeys. Exp. Brain Res. 135, $259-266$.

Hadjikhani, N., Liu, A.K., Dale, A.M., Cavanagh, P., Tootell, R.B., 1998. Retinotopy and color sensitivity in human visual cortical area V8. Nat. Neurosci. 1, 235-241.

Halgren, E., Dale, A.M., Sereno, M.I., Tootell, R.B., Marinkovic, K., Rosen, B.R., 1999. Location of human face-selective cortex with respect to retinotopic areas. Hum. Brain Mapp. 7, 29-37.

Heekeren, H.R., Marrett, S., Bandettini, P.A., Ungerleider, L.G., 2004. A general mechanism for perceptual decision-making in the human brain. Nature 431, 859-862.

Huk, A.C., Dougherty, R.F., Heeger, D.J., 2002. Retinotopy and functional subdivision of human areas MT and MST. J. Neurosci. 22, 7195-7205.

Koyama, M., Hasegawa, I., Osada, T., Adachi, Y., Nakahara, K., Miyashita, 
Y., 2004. Functional magnetic resonance imaging of macaque monkeys performing visually guided saccade tasks: comparison of cortical eye fields with humans. Neuron 41, 795-807.

Larsen, R.J., Marx, M.L., 1986. An Introduction to Mathematical Statistics and Its Applications, 2nd ed. Prentice-Hall, Englewood Cliffs, NJ.

Miller, E.K., Freedman, D.J., Wallis, J.D., 2002. The prefrontal cortex: categories, concepts and cognition. Philos. Trans. R. Soc. Lond., Ser. B Biol. Sci. 357, 1123-1136.

Owen, A.M., Evans, A.C., Petrides, M., 1996. Evidence for a two-stage model of spatial working memory processing within the lateral frontal cortex: a positron emission tomography study. Cereb. Cortex 6, 31-38.

Owen, A.M., Stern, C.E., Look, R.B., Tracey, I., Rosen, B.R., Petrides, M., 1998. Functional organization of spatial and nonspatial working memory processing within the human lateral frontal cortex. Proc. Natl. Acad. Sci. U. S. A. 95, 7721-7726.

Paus, T., 1996. Location and function of the human frontal eye-field: a selective review. Neuropsychologia 34, 475-483.

Phillips, P.J., Wechsler, H., Huang, J., Rauss, P., 1998. The FERET database and evaluation procedure for face recognition algorithms. Image Vis. Comput. J. 16, 295-306.

Rainer, G., Asaad, W.F., Miller, E.K., 1998. Memory fields of neurons in the primate prefrontal cortex. Proc. Natl. Acad. Sci. U. S. A. 95, $15008-15013$.

Reber, P.J., Wong, E.C., Buxton, R.B., Frank, L.R., 1998. Correction of off resonance-related distortion in echo-planar imaging using EPI-based field maps. Magn. Reson. Med. 39, 328-330.

Robinson, D.A., Fuchs, A.F., 1969. Eye movements evoked by stimulation of frontal eye fields. J. Neurophysiol. 32, 637-648.

Roe, A.W., Walled, D., Elzbieta, S., Goldman-Rakic, P.S., 2004. Optical imaging of prefrontal cortex during oculomotor delay response task in macaque monkey. Program No 1552004 Abstract Viewer/Itinerary Planner Washington, DC: Society for Neuroscience Online.

Rowe, J.B., Toni, I., Josephs, O., Frackowiak, R.S., Passingham, R.E., 2000. The prefrontal cortex: response selection or maintenance within working memory? Science 288, 1656-1660.

Sawaguchi, T., Iba, M., 2001. Prefrontal cortical representation of visuospatial working memory in monkeys examined by local inactivation with muscimol. J. Neurophysiol. 86, 2041-2053.

Sereno, M.I., Dale, A.M., Reppas, J.B., Kwong, K.K., Belliveau, J.W., Brady, T.J., Rosen, B.R., Tootell, R.B., 1995. Borders of multiple visual areas in humans revealed by functional magnetic resonance imaging. Science 268, 889-893.

Sereno, M.I., Pitzalis, S., Martinez, A., 2001. Mapping of contralateral space in retinotopic coordinates by a parietal cortical area in humans. Science 294, 1350-1354.

Simon, S.R., Meunier, M., Piettre, L., Berardi, A.M., Segebarth, C.M., Boussaoud, D., 2002. Spatial attention and memory versus motor preparation: premotor cortex involvement as revealed by fMRI. J. Neurophysiol. 88, 2047-2057.

Suzuki, H., Azuma, M., 1983. Topographic studies on visual neurons in the dorsolateral prefrontal cortex of the monkey. Exp. Brain Res. 53, 47-58.

Sweeney, J.A., Mintun, M.A., Kwee, S., Wiseman, M.B., Brown, D.L., Rosenberg, D.R., Carl, J.R., 1996. Positron emission tomography study of voluntary saccadic eye movements and spatial working memory. J. Neurophysiol. 75, 454-468.

Ward, B.D., 2000. Simultaneous inference for fMRI data. AFNI 3dDeconvolve Documentation, Medical College of Wisconsin.

Wojciulik, E., Kanwisher, N., Driver, J., 1998. Covert visual attention modulates face-specific activity in the human fusiform gyrus: fMRI study. J. Neurophysiol. 79, 1574-1578. 